Corrigendum

\title{
Corrigendum to "Diabetes Remission after Nonsurgical Intensive Lifestyle Intervention in Obese Patients with Type 2 Diabetes"
}

\author{
Adham Mottalib, Mahmoud Sakr, Mohamed Shehabeldin, and Osama Hamdy \\ Joslin Diabetes Center, One Joslin Place, Boston, MA 02215, USA \\ Correspondence should be addressed to Osama Hamdy; osama.hamdy@joslin.harvard.edu \\ Received 16 November 2015; Accepted 26 November 2015 \\ Copyright (C) 2016 Adham Mottalib et al. This is an open access article distributed under the Creative Commons Attribution \\ License, which permits unrestricted use, distribution, and reproduction in any medium, provided the original work is properly \\ cited.
}

Our paper titled "Diabetes Remission after Nonsurgical Intensive Lifestyle Intervention in Obese Patients with Type 2 Diabetes" [1] contains an error in Table 1. The body mass index (BMI) at 12 months was incorrectly reported as $40.0 \mathrm{~kg} / \mathrm{m}^{2}$ while the correct value is $34.0 \mathrm{~kg} / \mathrm{m}^{2}$. 
TABLE 1: Changes in cardiovascular risk factors after 12 weeks of intensive lifestyle intervention and at one year in participants who achieved major glycemic improvement and partial or complete diabetes remission.

\begin{tabular}{lccc}
\hline & Baseline & 3 months & 12 months \\
\hline Weight $(\mathrm{lbs})$ & $237.7 \pm 29.5$ & $211.3 \pm 26.1^{* *}$ & $211.1 \pm 26.5^{* *}$ \\
BMI $\left(\mathrm{kg} / \mathrm{m}^{2}\right)$ & $38.2 \pm 4$ & $34.0 \pm 3.8^{* *}$ & $34.0 \pm 4.2^{* *}$ \\
Systolic BP $(\mathrm{mmHg})$ & $128 \pm 11$ & $122 \pm 15^{*}$ & $124 \pm 16$ \\
Diastolic BP $(\mathrm{mmHg})$ & $73 \pm 8$ & $74 \pm 8$ & $73 \pm 9$ \\
Total cholesterol (mg/dL) & $171 \pm 31$ & $147 \pm 25^{* *}$ & $159 \pm 33$ \\
LDL-cholesterol (mg/dL) & $103 \pm 23$ & $88 \pm 18^{* *}$ & $91 \pm 24$ \\
HDL-cholesterol (mg/dL) & $41 \pm 10$ & $40 \pm 11$ & $47 \pm 13^{*}$ \\
Triglycerides $(\mathrm{mg} / \mathrm{dL})$ & $144 \pm 69$ & $94 \pm 41^{* *}$ & $123 \pm 42$ \\
\hline
\end{tabular}

$N=19$.

${ }^{*} p$ value $<0.05$ from baseline, ${ }^{* *} p$ value $<0.001$ from baseline.

\section{References}

[1] A. Mottalib, M. Sakr, M. Shehabeldin, and O. Hamdy, "Diabetes remission after nonsurgical intensive lifestyle intervention in obese patients with type 2 diabetes," Journal of Diabetes Research, vol. 2015, Article ID 468704, 4 pages, 2015. 


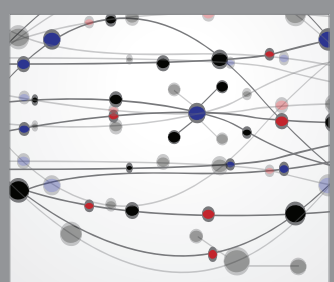

The Scientific World Journal
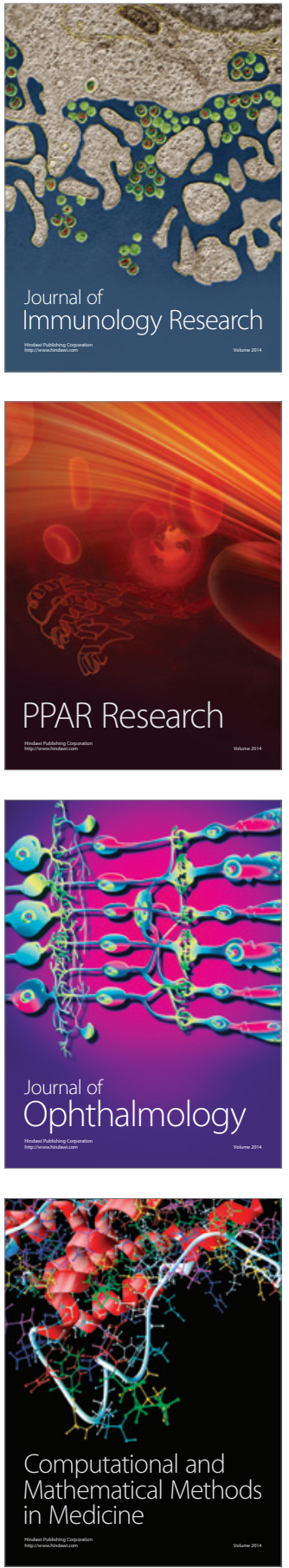

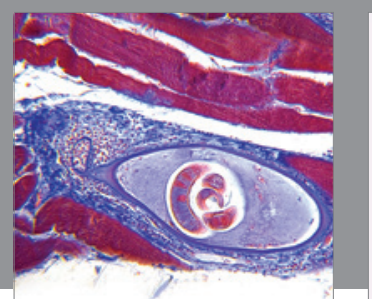

Gastroenterology Research and Practice

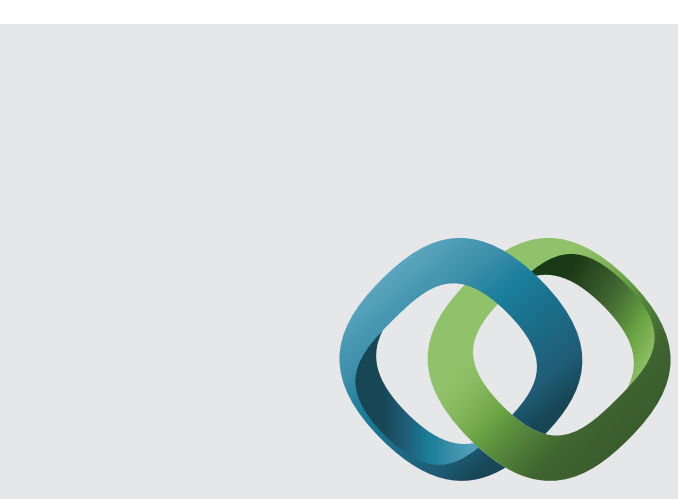

\section{Hindawi}

Submit your manuscripts at

http://www.hindawi.com
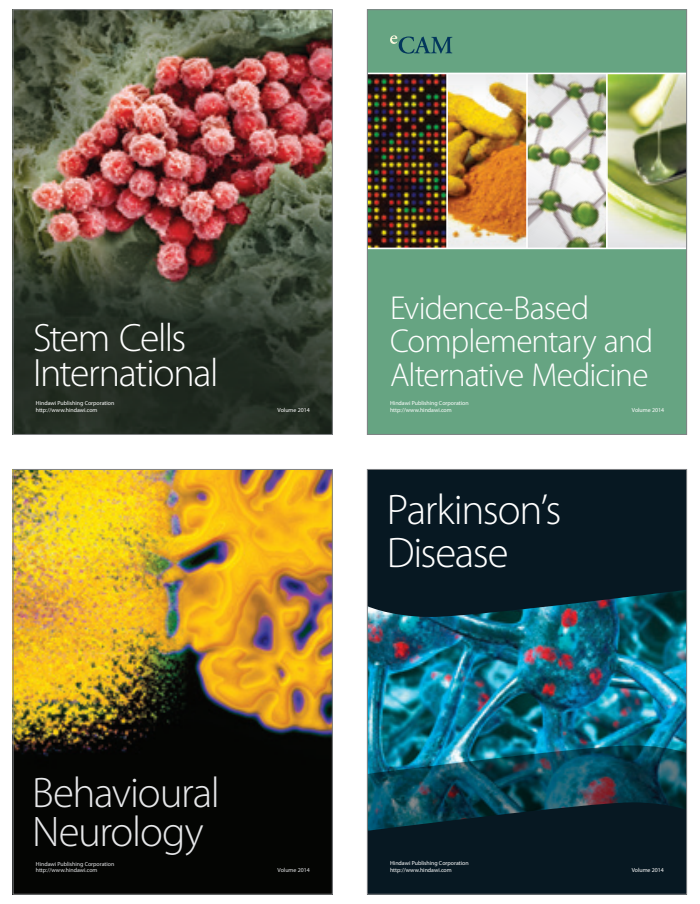
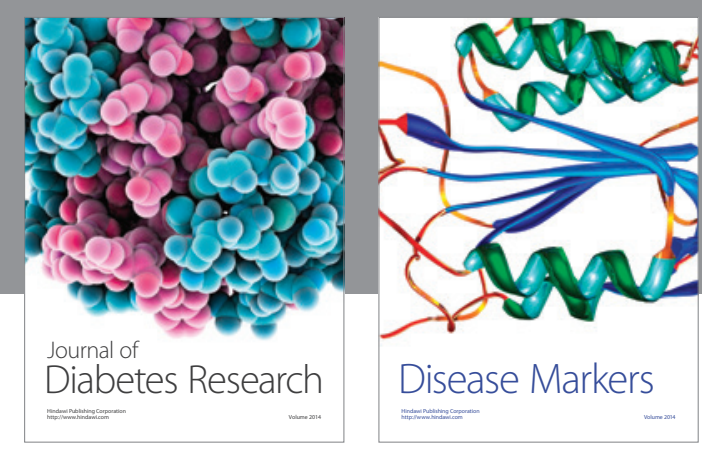

Disease Markers
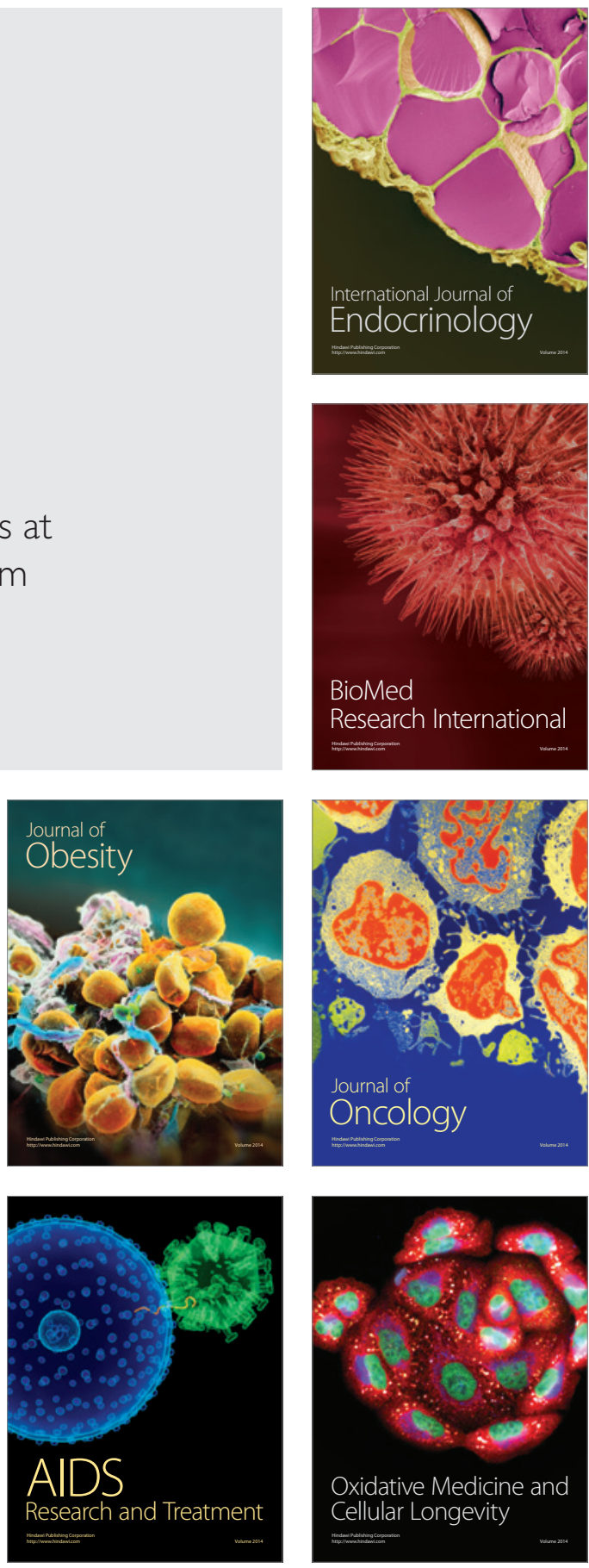